\title{
Rifampicin and Isoprodian in Combination in the Treatment of Leprosy
}

\author{
G. DEPASQUALE \\ St. Luke's Hospital, Department of Dermatology, \\ La Valetta, Malta
}

\begin{abstract}
Reviewing the progress over a 2 year period of 192 Maltese patients treated with rifampicin combined with Isoprodian, a much quicker response to therapy was observed than with any other type of therapy used earlier. The combination was effective even in cases previously treated for a number of years, though early cases seem to show the speediest response to treatment. The combination of drugs was well tolerated, and reactions could be controlled with the use of thalidomide without interruption of therapy. The therapy was most acceptable to patients.
\end{abstract}

Since the start of the Leprosy Eradication Programme in July 1972, the standard treatment of leprosy in Malta has been a combination of rifampicin and Isoprodian (= Prothionamide + isoniazid + dapsone).

One hundred and ninety-two patients, most of which had been treated previously for a number of years, were included in the programme from the start, while 18 other patients including 13 fresh cases were included at later stages. "Fresh cases" here refers to cases of recent diagnosis that have not received any previous treatment.

If one starts by studying the bacteriological results of this particular group of patients, one observes:

(a) there is a steady decrease in bacterial counts following treatment in practically all patients,

(b) as a general rule, the lower the initial count of bacteria the earlier is negativity reached,

(c) there seems to be no relation between age of the patients and their response to treatment.

Similar observations can be made on consideration of the large group of patients that were included in the programme from its start. There is great variation in the duration of treatment necessary before negativity in the sense of tissue clearance is reached. Even after 2 years of treatment we have a group of 20 patients who are at present not negative in this sense.

On detailed study one observes that as a general rule the longer standing the disease is, the longer the period of treatment required to achieve negativity. From the clinical point of view, the effects of therapy are seen earlier. Ulcerating 
nodules were observed to heal rapidly-sometimes in a matter of days after initiation of the treatment. The nodules themselves regressed at a later stage, while in some cases hard fibrotic nodules although decreased in size, did not disappear completely. Nasal obstruction and hoarseness were also relieved early in the course of treatment. Patients previously suffering from anaesthesia, reported marked improvement. Hair growth was observed in some patients who had been suffering from alopecia initially. Of particular importance is the fact that most patients reported that they were feeling physically much better after starting treatment.

Nausea and other gastric disturbances were reported initially by 44 patients (i.e. 20.9\%). However these side-effects subsided in almost all cases without interruption of therapy. Anaemia of moderate severity was recorded in 3 cases. In 1 of these cases the anaemia appeared 2 months after treatment had been discontinued. Mild anaemia occurred in 12 other cases. Jaundice appeared in 2 patients. Treatment was suspended until the jaundice cleared and it did not recur when treatment was restarted; according to our records serum bilirubin is slightly raised even in the untreated leprosy patients.

Lepra reactions occurred in 21 cases, mostly moderate to mild in severity. These reactions receded spontaneously or following the administration of thalidomide. Two patients suffered severe reactions requiring hospitalization. Corticosteroids were never used.

In general, we have observed a much quicker response to therapy with the use of rifampicin and Isoprodian than with any other type of therapy used earlier. The combination was effective even in cases previously treated for a number of years. However, early freshly diagnosed cases seemed to show a quicker response to treatment. The combination was well tolerated and reactions could be controlled with the use of thalidomide without interruption of therapy. Finally, the therapy was acceptable to the patients and they were very happy to carry on with the treatment as long as we advised it. 